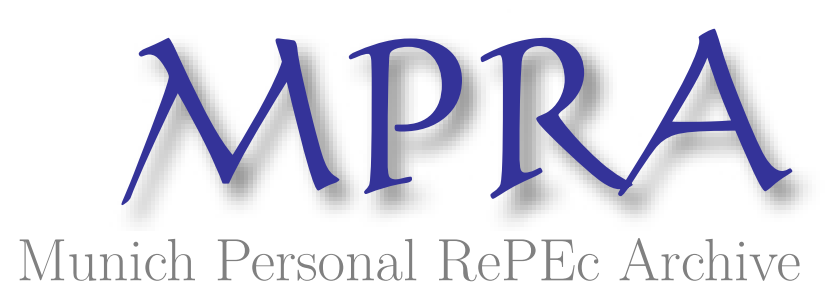

\title{
A Note on Almost Stochastic Dominance
}

Guo, Xu and Zhu, Xuehu and Wong, Wing-Keung and Zhu, Lixing

10 February 2013

Online at https://mpra.ub.uni-muenchen.de/44365/

MPRA Paper No. 44365, posted 14 Feb 2013 08:47 UTC 


\title{
A Note on Almost Stochastic Dominance
}

\author{
$\mathrm{Xu}$ Guo \\ Department of Mathematics, Hong Kong Baptist University \\ Xuehu Zhu \\ Department of Mathematics, Hong Kong Baptist University \\ Wing-Keung Wong \\ Department of Economics, Hong Kong Baptist University \\ Lixing Zhu \\ Department of Mathematics, Hong Kong Baptist University \\ February 10, 2013
}

\begin{abstract}
To satisfy the property of expected-utility maximization, Tzeng et al. (2012) modify the almost second-degree stochastic dominance proposed by Leshno and Levy (2002) and define almost higher-degree stochastic dominance. In this note, we further investigate the relevant properties. We define an almost third-degree stochastic dominance in the same way that Leshno and Levy (2002) define second-degree stochastic dominance and show that Leshno and Levy's (2002) almost stochastic dominance has the hierarchy property but not expected-utility maximization. In contrast, Tzeng et al.'s (2012) definition has the property of expected-utility maximization but not the hierarchy property. This phenomenon also holds for higher-degree stochastic dominance for these two concepts. Thus, the findings in this paper suggest that Leshno and Levy's (2002) definitions of ASSD and ATSD might be better than those defined by Tzeng et al. (2012) if the hierarchy property is considered to be an important issue.
\end{abstract}

Keywords : Almost stochastic dominance; expected-utility maximization; hierarchy of stochastic dominance.

Acknowledgement The authors thank Sally Burke for editing our paper. This research is partially supported by Hong Kong Baptist University and the Research Grants Council (RGC) of Hong Kong. 


\section{Introduction}

The theory of almost stochastic dominance (ASD) developed by Leshno and Levy (LL, 2002) plays an important role in several fields, particularly in financial research, and has drawn several important applications; see, for example, Levy (2006, 2009), Bali, et al. (2009), and Levy, et al. (2010). However, Tzeng et al. (THS, 2012) find an example that the almost second-degree stochastic dominance (ASSD) does not possess the property of expected-utility maximization. They modify the ASSD definition and show that the modified ASSD obtains the property of expected-utility maximization. It is well known that SD has the property of expected-utility maximization. However, it is also well known that SD has another important property - the property of hierarchy. In this paper we compare the performance of these two definitions of ASSD by investigating whether they both have the hierarchy property as the theory of standard stochastic dominance (SD) possesses.

In addition, in this paper we define the almost third-degree stochastic dominance (ATSD) in the same way that Leshno and Levy (2002) define ASSD and compare the performance of this ATSD with the ATSD used in Tzeng et al. (2012). Interestingly, we find that ASSD and ATSD as defined by Leshno and Levy (2002) or used the concept of Leshno and Levy (2002) possess the hierarchy property, while ASSD and ATSD as defined by Tzeng et al. (2012) do not. Thus, the findings in this paper suggest that Tzeng et al.'s (2012) definitions of ASSD and ATSD are not better than Leshno and Levy's (2002) definition of ASSD and the ATSD using the concept of Leshno and Levy (2002) if one considers possessing the hierarchy property to be an important issue.

\section{Notations and Definitions}

To describe the ASD concepts proposed by Leshno and Levy (2002) and Tzeng et al. (2012), we first state the definitions and notations to be used in this paper. Suppose that random variables $X$ and $Y$ defined on the support $\Omega=[\underline{x}, \bar{x}]$ have the corresponding distribution functions $F$ and $G$, respectively. The following notations will be used 
throughout this paper:

$$
\begin{aligned}
& H^{(1)}=H \text { and } H^{(n)}(x)=\int_{\underline{x}}^{x} H^{(n-1)}(t) d t \text { for } H=F, G \text { and } n=2,3 \\
& \left\|F^{(n)}-G^{(n)}\right\|=\int_{\underline{x}}^{\bar{x}}\left|F^{(n)}(x)-G^{(n)}(x)\right| d x \text { for } n=1,2,3 ; \\
& S_{1}(F, G)=\{x \in[\underline{x}, \bar{x}]: G(x)<F(x)\} ; \text { and } \\
& S_{n}(F, G)=\left\{x \in S_{n-1}(F, G): G^{(n)}(x)<F^{(n)}(x)\right\} \\
& \hat{S}_{n}(F, G)=\left\{x \in[\underline{x}, \bar{x}]: G^{(n)}(x)<F^{(n)}(x)\right\} \text { for } n=2,3 .
\end{aligned}
$$

An individual chooses between $F$ and $G$ in accordance with a consistent set of preferences satisfying the von Neumann-Morgenstern (1944) consistency properties. Accordingly, $F$ is preferred to $G$ if $E_{F}(u)-E_{G}(u) \geq 0$ where $E_{F}(u) \equiv \int_{\underline{x}}^{\bar{x}} u(x) d F(x)$ and $E_{G}(u) \equiv \int_{\underline{x}}^{\bar{x}} u(x) d G(x)$.

To distinguish the ASSD proposed by Leshno and Levy (2002) from that defined by Tzeng et al. (2012), in this paper we use $A S S D^{L L}$ and $A S S D^{T H S}$ to represent the ASSDs proposed by Leshno and Levy (2002) and Tzeng et al. (2012), respectively. In addition, we use $A T S D^{L L}$ to indicate ATSD that we define in the same way that Leshno and Levy (2002) define ASSD and we use $A T S D^{T H S}$ to correspond to Tzeng et al.'s (2012) definition. We first state the definitions of the almost first-degree stochastic dominance (AFSD) and $A S S D^{L L}$ defined by Leshno and Levy (2002) as follows:

Definition 2.1 For $0<\epsilon<1 / 2$,

AFSD: $F$ is said to dominate $G$ by $\epsilon-A F S D$, denoted by $F \succeq_{1}^{\text {almost }(\epsilon)} G$, if and only if

$$
\int_{S_{1}}[F(x)-G(x)] d x \leq \epsilon\|F-G\|, \text { and }
$$

$A S S D^{L L}: F$ is said to dominate $G$ by $\epsilon-A S S D^{L L}$, denoted by $F \succeq_{2^{L L}}^{\text {almost }(\epsilon)} G$, if and only if

$$
\int_{S_{2}}[F(x)-G(x)] d x \leq \epsilon\|F-G\| \text { and } E_{F}(X) \geq E_{G}(X)
$$

where $S_{n}=S_{n}(F, G)$ for $n=1,2$ and $\|F-G\|$ are defined in (2.1).

Tzeng et al. (2012) define $A S S D^{T H S}$ and $A T S D^{T H S}$ as follows: 
Definition 2.2 For $0<\epsilon<1 / 2$,

$A S S D^{T H S}: F$ is said to dominate $G$ by $\epsilon$-ASS $D^{T H S}$, denoted by $F \succeq_{2^{T H S}}^{\text {almost }(\epsilon)} G$, if and only if

$$
\int_{\hat{S}_{2}}\left[F^{(2)}(x)-G^{(2)}(x)\right] d x \leq \epsilon\left\|F^{(2)}-G^{(2)}\right\| \quad \text { and } \quad E_{F}(X) \geq E_{G}(X),
$$

$A T S D^{T H S}: F$ is said to dominate $G$ by $\epsilon$-ATS $D^{T H S}$, denoted by $F \succeq_{3^{T H S}}^{\text {almost }(\epsilon)} G$, if and only if

$$
\int_{\hat{S}_{3}}\left[F^{(3)}(x)-G^{(3)}(x)\right] d x \leq \epsilon|| F^{(3)}-G^{(3)} \| \quad \text { and } G^{(n)}(\bar{x})-F^{(n)}(\bar{x}) \geq 0 \text { for } n=2,3
$$

where $\hat{S}_{n}=\hat{S}_{n}(F, G), F^{(n)}, G^{(n)}$, and $\left\|F^{(n)}-G^{(n)}\right\|$ are defined in (2.1) for $n=2$ and 3 .

In this note, we define $A T S D^{L L}$ in the same way that Leshno and Levy (2002) define $A S S D^{L L}$ as shown in the following:

Definition 2.3 For $0<\epsilon<1 / 2$,

$A T S D^{L L}: F$ is said to dominate $G$ by $\epsilon-A T S D^{L L}$, denoted by $F \succeq_{3^{L L}}^{\text {almost }(\epsilon)} G$, if and only if

$$
\int_{S_{3}}[F(x)-G(x)] d x \leq \epsilon\|F-G\| \text { and } E_{F}(X) \geq E_{G}(X),
$$

where $S_{3}=S_{3}(F, G)$ and $\|F-G\|$ are defined in (2.1).

Remark 2.1 We note that if one incorporates the idea of Definition 2.2 into Definition 2.1, one may suggest replacing the conditions stated in (2.6) with the following conditions for $A S S D^{L L}$ :

$$
\int_{S_{3}}[F(x)-G(x)] d x \leq \epsilon|| F-G \| \text { and } G^{(n)}(\bar{x})-F^{(n)}(\bar{x}) \geq 0 \text { for } n=2,3 .
$$

However, because $E_{F}(X)-E_{G}(X)=G^{(2)}(\bar{x})-F^{(2)}(\bar{x})$, conditions stated in equation (2.7) add condition $G^{(3)}(\bar{x})-F^{(3)}(\bar{x}) \geq 0$ to equation (2.6). Nevertheless, if conditions stated in equation (2.7) are used, the property of hierarchy will not hold. For example, consider the example discussed in Appendix A; one can easily show that $F$ dominates $G$ by $A S S D^{L L}$. On the other hand, by using this example, we have $G^{(3)}(1)=\frac{1}{2}\left(1-\frac{1}{3}\right)^{2}=$ $\frac{2}{9}<F^{(3)}(1)=\frac{1}{4}$, and thus, the property of hierarchy does not hold. In this connection, in this paper we will only use Definition 2.3 with the conditions stated in equation (2.6) to define $A T S D^{L L}$. 
Before we continue our discussion, we first state the sets of utility functions as shown in the following definition:

Definition 2.4 For $n=1,2$ and 3, $u \in U_{n}$ or $U_{n}^{*}(\epsilon)$ is a utility function such that

$$
\begin{aligned}
U_{n} & =\left\{u:(-1)^{i} u^{(i)} \leq 0, i=1, \cdots, n\right\} \text { and } \\
U_{n}^{*}(\epsilon) & =\left\{u \in U_{n}:(-1)^{n+1} u^{(n)}(x) \leq \inf \left\{(-1)^{n+1} u^{(n)}(x)\right\}[1 / \epsilon-1] \forall x\right\} .
\end{aligned}
$$

\section{The Theory}

\subsection{Expected-Utility Maximization}

The stochastic dominance approach is regarded as one of the most useful tools for ranking investment prospects when there is uncertainty because it possesses the property of expected-utility maximization that ranking assets by FSD, SSD, and TSD is equivalent to expected-utility maximization for investors with utility functions $u \in U_{1}, U_{2}$, and $U_{3}$, respectively (Hadar and Russell, 1969; Whitmore, 1970).

It is interesting to examine whether almost SD possesses a property of expectedutility maximization similar to SD. Leshno and Levy (2002) show that ranking assets by AFSD is equivalent to expected-utility maximization for investors with utility functions $u \in U_{1}^{*}(\epsilon)$. Nevertheless, Tzeng et al. (2012) show that ranking assets by $A S S D^{L L}$ is not equivalent to expected-utility maximization for investors with utility functions $u \in U_{2}^{*}(\epsilon)$. They then define $A S S D^{T H S}$ and show that, on the contrary, ranking assets by $A S S D^{T H S}$ is equivalent to expected-utility maximization for investors with utility functions $u \in$ $U_{2}^{*}(\epsilon)$. In addition, they define $A n S D^{T H S}$ and show that ranking assets by $A n S D^{T H S}$ is equivalent to expected-utility maximization for investors with utility functions $u \in$ $U_{n}^{*}(\epsilon)$ for $n>2$. Thus, the first aim of this paper is to examine whether ranking assets by $A T S D^{L L}$, the newly defined ATSD in this paper, is equivalent to expected-utility maximization for investors with utility functions $u \in U_{3}^{*}(\epsilon)$. Together with the finding from Tzeng et al. (2012), we state the property of non-expected-utility maximization for both $A S S D^{L L}$ and $A T S D^{L L}$ in the following property: 
Property 3.1 (Non-Expected-Utility Maximization of both $A S S D^{L L}$ and $A T S D^{L L}$ )

1. There exists utility function $u \in U_{2}^{*}(\epsilon)$ such that $E_{F}(u)<E_{G}(u)$ even when $F$ dominates $G$ by $\epsilon-A S S D^{L L}\left(F \succeq_{2^{L L}}^{\text {almost }(\epsilon)} G\right)$, for some $\epsilon<1 / 2$.

2. There exists utility function $u \in U_{3}^{*}(\epsilon)$ such that $E_{F}(u)<E_{G}(u)$ even when $F$ dominates $G$ by $\epsilon$-ATS $D^{L L}\left(F \succeq_{3^{L L}}^{\text {almost }(\epsilon)} G\right)$ for some $\epsilon<1 / 2$.

Readers may refer to Tzeng et al. (2012) for the example being constructed to show Part 1 of Property 3.1. In this paper we will construct an example to show Part 2 of Property 3.1 in Appendix A.

\subsection{Hierarchy}

It is well known that SD possesses the expected-utility maximization property. However, SD also has another important property - the property of hierarchy - that FSD implies SSD, which, in turn, implies TSD. In this paper, we discover that the $A n S D^{T H S}$ introduced by THS does not possess the property of hierarchy as stated in the following property for $n=1,2$, and $3:^{1}$

\section{Property 3.2 (Non-Hierarchy of $A n S D^{T H S}$ )}

1. If $F$ dominates $G$ by $\epsilon$-AFSD $\left(F \succeq_{1}^{\text {almost }(\epsilon)} G\right)$ for some $\epsilon<1 / 2$, it is not necessary that $F$ dominates $G$ by $\epsilon$-ASSD $D^{T H S}\left(F \succeq_{2^{T H S}}^{\text {almost }(\epsilon)} G\right)$ for some $\epsilon<1 / 2$.

2. If $F$ dominates $G$ by $\epsilon$-ASSD $D^{T H S}\left(F \succeq_{2^{T H S}}^{\text {almost }(\epsilon)} G\right)$ for some $\epsilon<1 / 2$, it is not necessary that $F$ dominates $G$ by $\epsilon$-ATS $D^{T H S}\left(F \succeq_{2^{T H S}}^{\text {almost }(\epsilon)} G\right)$ for some $\epsilon<1 / 2$.

We will construct two examples for Property 3.2 in Appendix B. Nonetheless, we find that $A S S D^{L L}$ possesses the property of hierarchy as shown in the following theorem:

\section{Theorem 3.1 (Hierarchy of $A n S D^{L L}$ )}

\footnotetext{
${ }^{1}$ We note that one could easily extend our work to $n>3$. However, though some studies, see, for example, Eeckhoudt and Schlesinger (2006) and Denuit and Eeckhoudt (2010), study risk to $n>3$, most academics and practitioners are only interested in studying the case up to $n=3$. Thus, we stop at $n=3$.
} 
1. If $F$ dominates $G$ by $\epsilon$-AFSD $\left(F \succeq_{1}^{\text {almost }(\epsilon)} G\right)$ for some $\epsilon<1 / 2$, then $F$ will dominate $G$ by $\epsilon$-ASS $D^{L L}\left(F \succeq_{2^{L L}}^{\text {almost }(\epsilon)} G\right)$ for some $\epsilon<1 / 2$.

2. If $F$ dominates $G$ by $\epsilon$-ASSD $D^{L L}\left(F \succeq_{2^{L L}}^{\text {almost }(\epsilon)} G\right)$ for some $\epsilon<1 / 2$, then $F$ will dominate $G$ by $\epsilon$-ATS $D^{L L}\left(F \succeq_{3^{L L}}^{\text {almost }(\epsilon)} G\right)$ for some $\epsilon<1 / 2$.

\section{Concluding Remarks}

The findings in this paper lead us to conclude that if expected-utility maximization is used to measure ASD, $A n S D^{T H S}$ is preferred to $A n S D^{L L}$. However, when hierarchy is considered, $A n S D^{L L}$ is preferred.

\section{Appendix}

\section{Appendix A. An example for Part 2 of Property 3.1:}

Let $x \in[0,1]$. Assume that there are two payoff distributions such that

$$
F(x)=\left\{\begin{array}{ll}
\frac{1}{2} & \text { if } 0 \leq x<1 \\
1 & \text { if } x=1
\end{array} \text { and } G(x)= \begin{cases}0 & \text { if } 0<x<\frac{1}{3} \\
1 & \text { if } \frac{1}{3} \leq x\end{cases}\right.
$$

Figure A.1 exhibits the plots of $F$ and $G$. In Appendix B, we show that $F$ dominates $G$ by $\epsilon$-AFSD. According to Theorem 3.1, one could easily find that $F$ dominates $G$ by $\epsilon$-ATSD $D^{L L}\left(F \succeq_{3^{L L}}^{\text {almost }(\epsilon)} G\right)$. Nevertheless, one could easily find an investor with a utility function $u \in U_{3}^{*}(\epsilon)$ who strictly prefers $G$ to $F$; i.e, $E_{F}(u)<E_{G}(u)$. Here, we suggest one as follows:

$$
u(x)=x^{3}-3.5 x^{2}+5 x \quad \text { with } \quad 0 \leq x \leq 1
$$

Some simple computations can show that $u$ belongs to $U_{3}^{*}(\epsilon)$ for all $0<\epsilon<1 / 2$. On the other hand, we can have $E_{F}(u)=u(0) \frac{1}{2}+u(1) \frac{1}{2}=1.25$ and $E_{G}(u)=u\left(\frac{1}{3}\right)=1.3148>$ $E_{F}(u)$. This confirms that the assertions in Property 3.1 hold. 


\section{Appendix B. Examples for Property 3.2:}

Construct an example for part 1 of Property 3.2. We use the same distributions $F$ and $G$ as those in (A.1). We have $E_{F}(X)=1 / 2$ and $E_{G}(X)=1 / 3$ and obtain the following

$$
\begin{aligned}
& F^{(2)}(x)=\left\{\begin{array}{ll}
\frac{1}{2} x & \text { if } 0 \leq x<1, \\
\frac{1}{2} & \text { if } x=1,
\end{array}, G^{(2)}(x)=\left\{\begin{array}{ll}
0 & \text { if } 0<x<\frac{1}{3}, \\
x-\frac{1}{3} & \text { if } \frac{1}{3} \leq x,
\end{array},\right.\right. \\
& S_{1}(F, G)=\{x: G(x)<F(x)\}=\{x: x \in[0,1 / 3]\}, \\
& \hat{S}_{2}(F, G)=\left\{x: G^{(2)}(x)<F^{(2)}(x)\right\}=\{x: x \in[0,2 / 3]\} .
\end{aligned}
$$

Thus, according to Definition 2.1 for the AFSD, it is obvious that $F$ dominates $G$ by $\epsilon$-AFSD with, e.g.

$$
\epsilon \geq \frac{\int_{S_{1}}[F(x)-G(x)] d x}{\|F-G\|}=\frac{1 / 6}{1 / 2}=1 / 3 .
$$

However, there is no $A S S D^{T H S}$ as shown in the following:

$$
\begin{aligned}
\int_{\hat{S}_{2}}\left[F^{(2)}(x)-G^{(2)}(x)\right] d x & =\int_{0}^{\frac{1}{3}} \frac{1}{2} x d x+\int_{\frac{1}{3}}^{\frac{2}{3}}\left(\frac{1}{2} x-x+\frac{1}{3}\right) d x \\
& =\frac{1}{4} \times \frac{1}{9}+\frac{1}{3} \times \frac{1}{3}-\frac{1}{4}\left(\frac{4}{9}-\frac{1}{9}\right)=\frac{1}{18}
\end{aligned}
$$

and

$$
\begin{aligned}
\int_{\hat{S}_{2}^{C}}\left[G^{(2)}(x)-F^{(2)}(x)\right] d x & =\int_{\frac{2}{3}}^{1}\left(x-\frac{1}{3}-\frac{1}{2} x\right) d x \\
& =\frac{1}{4} \times\left(1-\frac{4}{9}\right)-\frac{1}{3} \times \frac{1}{3}=\frac{1}{36}
\end{aligned}
$$

in which $\hat{S}_{2}^{C}$ is a complement of $\hat{S}_{2}$. As a result, we have

$$
\int_{\hat{S}_{2}}\left[F^{(2)}(x)-G^{(2)}(x)\right] d x=\frac{2}{3}\left\|F^{(2)}-G^{(2)}\right\|,
$$

which implies that $F$ does not dominate $G$ by the $A S S D^{T H S}$. This confirms Part 1 of Property 3.2.

We turn to constructing an example for part 2 of Property 3.2. Let $x \in[0,1]$ and we choose $F$ from (A.1) and select $G$ to be

$$
G(x)=\left\{\begin{array}{ll}
0 & \text { if } 0<x<\frac{1}{4} \\
1 & \text { if } \frac{1}{4} \leq x
\end{array} .\right.
$$


Then, we have $E_{F}(X)=1 / 2$ and $E_{G}(X)=1 / 4$ and obtain the following:

$$
\begin{aligned}
& G^{(2)}(x)=\left\{\begin{array}{ll}
0 & \text { if } 0<x<\frac{1}{4} \\
x-\frac{1}{4} & \text { if } \frac{1}{4} \leq x
\end{array} \quad, \quad F^{(3)}(x)= \begin{cases}\frac{1}{4} x^{2} & \text { if } 0 \leq x<1 \\
\frac{1}{4} & \text { if } x=1\end{cases} \right. \\
& G^{(3)}(x)=\left\{\begin{array}{ll}
0 & \text { if } 0<x<\frac{1}{4} \\
\frac{1}{2}\left(x-\frac{1}{4}\right)^{2} & \text { if } \frac{1}{4} \leq x
\end{array},\right. \\
& S_{1}(F, G)=\{x: G(x)<F(x)\}=\{x: x \in[0,1 / 4]\}, \\
& \hat{S}_{2}(F, G)=\left\{x: G^{(2)}(x)<F^{(2)}(x)\right\}=\{x: x \in[0,1 / 2]\}, \\
& \hat{S}_{3}(F, G)=\left\{x: G^{(3)}(x)<F^{(3)}(x)\right\}=\{x: x \in[0,(2+\sqrt{2}) / 4]\} .
\end{aligned}
$$

We are now ready to show that $F$ dominates $G$ by the $A S S D^{T H S}$. Note that we can have

$$
\int_{\hat{S}_{2}}\left[F^{(2)}(x)-G^{(2)}(x)\right] d x=\int_{0}^{\frac{1}{4}} \frac{1}{2} x d x+\int_{\frac{1}{4}}^{\frac{2}{4}}\left(\frac{1}{2} x-x+\frac{1}{4}\right) d x=\frac{1}{32}
$$

and

$$
\int_{\hat{S}_{2}^{C}}\left[G^{(2)}(x)-F^{(2)}(x)\right] d x=\int_{\frac{2}{4}}^{1}\left(x-\frac{1}{4}-\frac{1}{2} x\right) d x=\frac{1}{16} .
$$

As a result, we get

$$
\int_{\hat{S}_{2}}\left[F^{(2)}(x)-G^{(2)}(x)\right] d x=\frac{1}{3}\left\|F^{(2)}-G^{(2)}\right\|,
$$

and thus, $F$ dominates $G$ by the $A S S D^{T H S}$.

On the other hand, though we can have $G^{(2)}(1)=3 / 4>1 / 2=F^{(2)}(1)$ and $G^{(3)}(1)=$ $9 / 32>1 / 4=F^{(3)}(1), F$ does not dominate $G$ by the $A T S D^{T H S}$. We show this argument in the following. We first obtain

$$
\begin{aligned}
\int_{\hat{S}_{3}}\left[F^{(3)}(x)-G^{(2)}(x)\right] d x & =\int_{0}^{\frac{1}{4}} \frac{1}{4} x^{2} d x+\int_{\frac{1}{4}}^{\frac{2+\sqrt{2}}{4}}\left(\frac{1}{4} x^{2}-\frac{1}{2}\left(x-\frac{1}{4}\right)^{2}\right) d x \\
& =0.0152
\end{aligned}
$$

and

$$
\begin{aligned}
\int_{\hat{S}_{3}^{C}}\left[G^{(3)}(x)-F^{(3)}(x)\right] d x & =\int_{\frac{2+\sqrt{2}}{4}}^{1}\left(\frac{1}{2}\left(x-\frac{1}{4}\right)^{2}-\frac{1}{4} x^{2}\right) d x \\
& =0.0022 .
\end{aligned}
$$

Thus, we get

$$
\int_{\hat{S}_{3}}\left[F^{(3)}(x)-G^{(3)}(x)\right] d x>\frac{1}{2}\left\|F^{(3)}-G^{(3)}\right\|,
$$

which implies that $F$ does not dominate $G$ by the $A T S D^{T H S}$ and confirm the assertion in Property 3.2. 


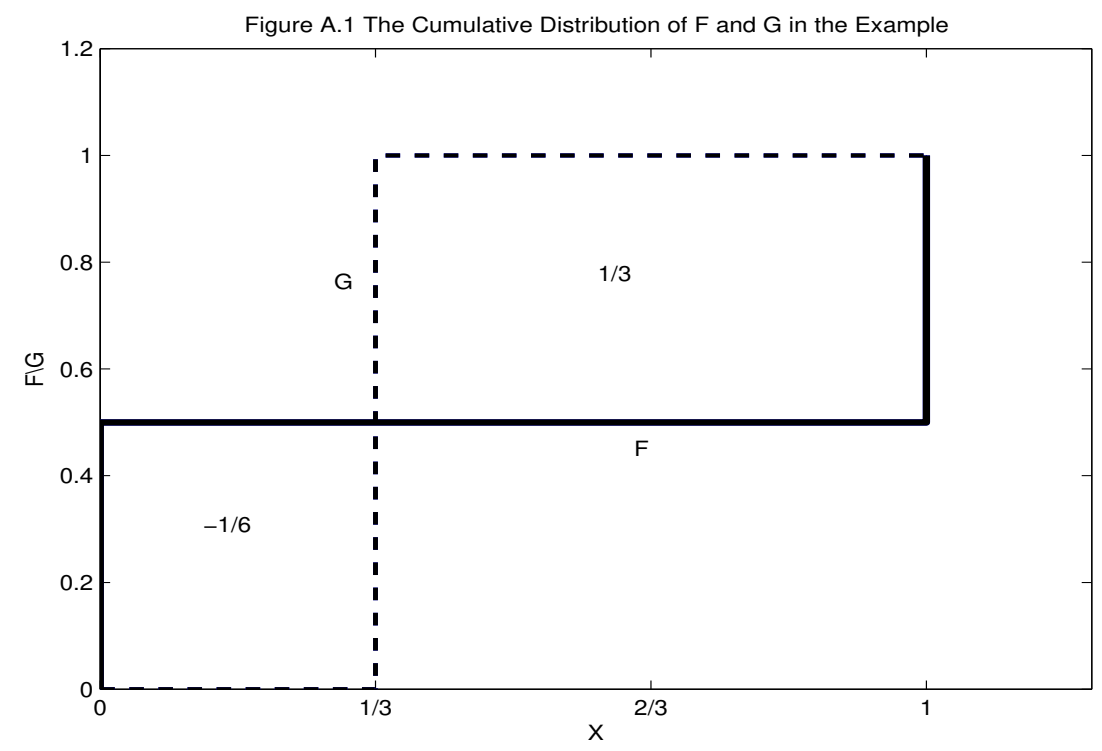

Figure A.2 $F^{(2)}$ and $G^{(2)}$ in the Example

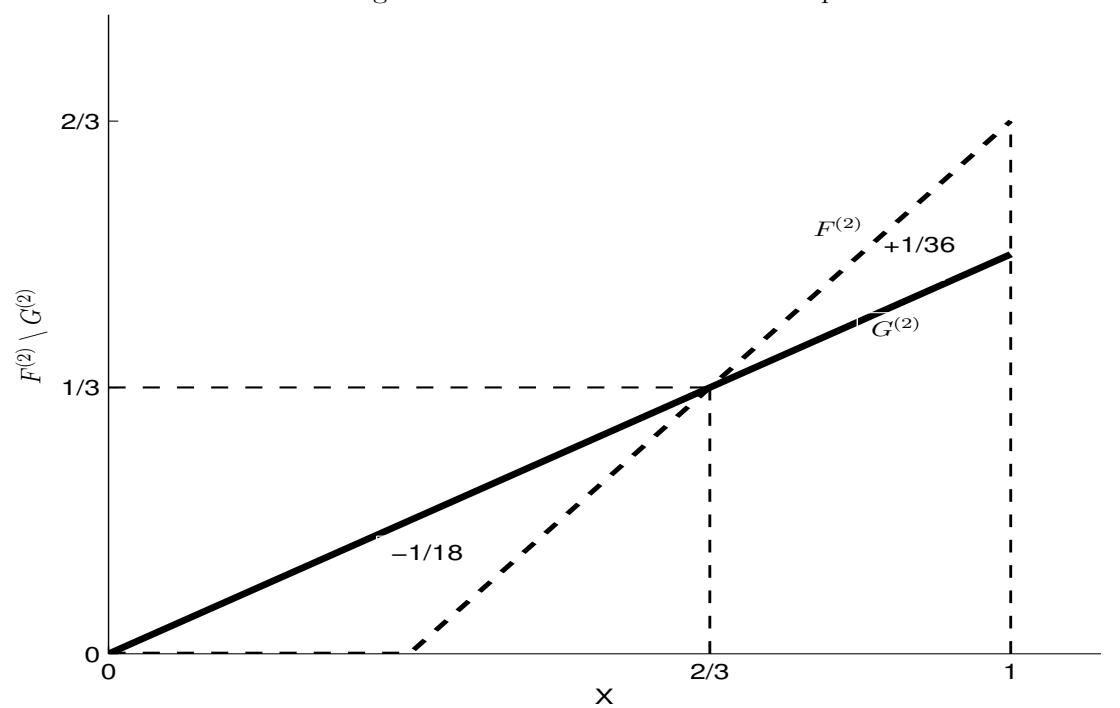




\section{References}

Bali, T.G., Demirtas, K. O., Levy, H., Wolf, A. (2009), Bonds versus stocks: Investors' age and risk taking. Journal of Monetary Economics, 56(6), 817-830.

Denuit, M. M., Eeckhoudt, L. (2010), A general index of absolute risk attitude. Management Science, 56(4), 712-715.

Eeckhoudt, L., Schlesinger, H. (2006), Putting risk in its proper place. American Economic Review, 96(1), 280-289.

Hadar J., Russell W.R. (1969), Rules for Ordering Uncertain Prospects, American Economic Review, 59, 25-34.

Leshno, M., Levy, H. (2002), Preferred by "all" and preferred by "most" decision makers: Almost stochastic dominance. Management Science, 48(8), 1074-1085.

Levy, H. (2006), Stochastic Dominance: Investment Decision Making Under Uncertainty, Springer, New York.

Levy, M. (2009), Almost Stochastic Dominance and stocks for the long run, European Journal of Operational Research 194, 250-257.

Levy, H., Leshno, M, Leibovitch, B. (2010), Economically relevant preferences for all observed epsilon, Annals of Operations Research, 176, 153-178.

Tzeng, L. Y., Huang, R. J., Shih, P-T. (2012), Revisiting Almost Second-Degree Stochastic Dominance, Management Science, DOI:10.1287/mnsc.1120.1616.

von Neumann, J., Morgenstern, O., 1944, Theory of Games and Economic Behavior. Princeton University Press, Princeton N.J.

Whitmore G.A. (1970), Third-degree Stochastic Dominance, American Economic Review, 60, 457-459. 